hep-ph/0610098

October 9, 2006

\title{
Top quark signatures of Higgsless models ${ }^{1}$
}

\author{
Christian Schwinn \\ Institut für Theoretische Physik E, RWTH Aachen, D - 52056 Aachen, Germany
}

\begin{abstract}
In these proceedings I describe the use of tree level unitarity to constrain top quark signatures of Higgsless electroweak models and discuss implications for collider phenomenology.
\end{abstract}

Keywords: Electroweak symmetry breaking, Top quarks, Extra dimensions

PACS: 12.15.-y, 14.65.,11.10.Kk

\section{HIGGSLESS ELECTROWEAK SYMMETRY BREAKING}

Recently higher dimensional Higgsless electroweak models have been proposed where the gauge symmetry is broken by boundary conditions [1] and the bad high energy behavior of $W$ and $Z$ scattering amplitudes is softened by the exchange of $W$ and $Z$ Kaluza-Klein (KK)-modes instead of that of a Higgs boson [1, 2, 3]. Implementations of this idea employ e.g. a $S U(2)_{L} \otimes S U(2)_{R} \otimes U(1)$ in a warped extra dimension [4, 5], a simple $S U(2)$ in a flat extra dimension [6] or four dimensional $S U(2)^{N} \otimes U(1)^{M}$ 'theory spaces' [7,8]. Fermion masses in 5D Higgsless models can be generated by gauge invariant brane localized mass and kinetic terms [4] without violating the relevant unitarity sum rules (SRs) [9]. Electroweak precision data can be satisfied by delocalizing the zero modes of light fermions and choosing appropriate bulk masses $[4,7,6]$.

Some signatures of Higgsless models-e.g. Drell-Yan production of KK-gauge bosons-depend on details of the light fermion sector. In contrast, narrow KKresonances below $1 \mathrm{TeV}$ in vector boson fusion [10] and anomalous gauge boson couplings $[1,7]$ have been identified as a generic prediction of the Higgsless mechanism. We will briefly review the argument to prepare for an analogous approach to top signatures [11] in the next section. Following [10], one can use the unitarity sum rules ensuring the cancellation of terms growing like $s^{2}$ and $s$ in $W Z \rightarrow W Z$ scattering

$$
g_{W^{2} Z^{2}}-g_{W W Z}^{2}=\sum_{n} g_{Z W W^{(n)}}^{2} \quad g_{Z W W^{2}}^{2} m_{Z}^{4}=3 m_{W}^{2} \sum_{n} g_{Z W W^{(n)}}^{2} m_{W^{(n)}}^{2}
$$

to estimate the coupling of the first $W$-KK-mode as $g_{Z W W^{(1)}} \lesssim\left(g m_{Z}\right) /\left(\sqrt{3} m_{W^{(1)}}\right)$ which has found to be promising for a detection at the LHC. Since the Standard model (SM) couplings alone satisfy the first SR in (1), Higgsless models also require anomalous gauge boson couplings [1]. Using (1), one estimates $\delta g_{W W Z} / g_{W W Z}=c \times\left(m_{W} / m_{W^{(1)}}\right)^{2}$ with $c \sim \mathscr{O}(1)$ in agreement with the result [7] $c=\pi^{2} /\left(12 \cos \theta_{W}^{2}\right)$ in a flat 5D model.

\footnotetext{
1 To appear in the proceedings of SUSY06, 14th International Conference on Supersymmetry and the Unification of Fundamental Interactions Irvine, California, USA 12-17 June 2006
} 


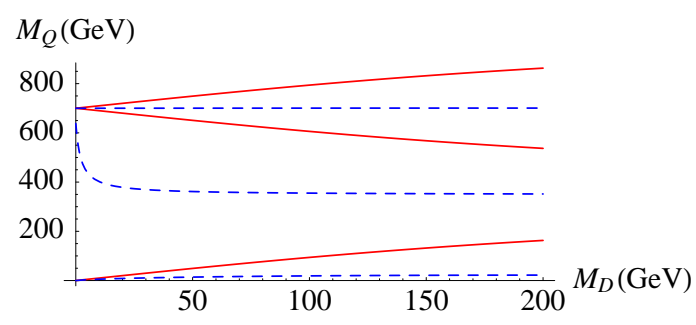

FIGURE 1. Masses of bottom (blue/dashed) and top (red/solid) zero- and KK-modes as function of the boundary mass parameter $M_{D}$ in a flat space $S U(2)_{L} \otimes S U(2)_{R} \otimes U(1)$ toy model for $R^{-1}=700 \mathrm{GeV}$

The large mass of the top quark implies a special role for the third family. As an example, figure 1 shows the mass spectrum in a flat space $S U(2)_{L} \otimes S U(2)_{R} \otimes U(1)$ toy-model where the $t$ and $b$ quarks are embedded into bulk multiplets $Q_{L}=\left(t_{L}, b_{R}\right)$ and $Q_{R}=\left(t_{R}, b_{R}\right)$ as in [4]. One the brane at $y=\pi R$ the symmetry breaking pattern $S U(2)_{L} \otimes S U(2)_{R} \rightarrow S U(2)_{V}$ allows a localized mass term $\delta(y-\pi R) M_{D}\left(\bar{Q}_{L} Q_{R}+\right.$ $\left.\bar{Q}_{R} Q_{L}\right)$. The large $M_{D}$ needed for the top mass splits the KK-modes of $Q_{L}$ and $Q_{R}$ leading to $m_{T^{(1)}}, m_{B^{(1)}}<m_{Z^{(1)}}$. Similar results are found in a warped model [5]. For the embedding of $b_{R}$ chosen in this example, the bottom-top mass splitting requires a large boundary kinetic term (BKT) term for $b_{R}$ at the $y=0$ brane where $S U(2)_{R}$ is broken, further reducing $m_{B^{(1)}}$. In such a setup, it has been found difficult to obtain a large enough top quark mass while respecting bounds on anomalous $Z \bar{b}_{L} b_{L}$ or $W \bar{t}_{R} b_{R}$ couplings $[5,4,6]$.

Two strategies have been proposed to overcome this difficulties: a): raising the mass of the $t$ and $b$ KK-modes, e.g. by introducing two slices of warped spaces with different curvatures [12], by allowing 5D Lorentz invariance violating bulk-kinetic terms [6], or using theory space models [8]. b): protecting the $Z b b$ vertex by using a symmetric representation of $S U(2)_{L} \otimes S U(2)_{R}$ for the $5 D$ multiplet containing $b_{L}[13,14]$. Many third family signatures of Higgsless models depend on such model building choices, e.g. the strongest constraints from anomalous couplings can arise from $Z \bar{b}_{L} b_{L}$ [4] or $W \bar{t}_{R} b_{R}$ [6] while large anomalous $Z \bar{t} t$ couplings arise in [14]. The lower bound on $m_{T^{(1)}}$ ranges from $450 \mathrm{GeV}$ in [14] to $1.6 \mathrm{TeV}$ [6]. Finally, the two-bulk model of [12] features additional scalar top-pions while the mechanism of $[13,14]$ involves KK-modes of colored fermions with $Q=\frac{5}{3}$.

\section{UNITARITY CONSTRAINTS ON TOP SIGNATURES}

As in the gauge boson sector [10], unitarity SRs can be used to constrain signatures of the Higgsless mechanism in the top sector [11]. In the 4D SM without a Higgs, the scattering amplitude for $W^{+} W^{-} \rightarrow t \bar{t}$ grows like $\sqrt{s}$. In 5D Higgsless models the cancellation of this growth by the exchange of $Z$ and bottom KK-modes requires the SRs $[9,11,6]$

$$
\begin{gathered}
-\left(g_{W t b}^{L / R}\right)^{2}+g_{Z W W} g_{t t Z}^{L / R}+g_{W W \gamma} g_{t t \gamma}=\sum_{n}\left[\left(g_{W t B^{(n)}}^{L / R}\right)^{2}-g_{W W Z^{(n)}} g_{t t Z^{(n)}}^{L / R}\right]=0 \\
\left(g_{W t b}^{L}\right)^{2}+\left(g_{W t b}^{R}\right)^{2}=\sum_{n}\left[2 \frac{m_{B^{(n)}}}{m_{t}} g_{W t B^{(n)}}^{R} g_{W t B^{(n)}}^{L}-\left(g_{W t B^{(n)}}^{L}\right)^{2}-\left(g_{W t B^{(n)}}^{R}\right)^{2}\right]
\end{gathered}
$$


Since the Z-KK-modes do not appear in (2b), the unitarity cancellations cannot be achieved by including only vector boson resonances and the presence of the third family quark KK-modes is a generic consequence of the Higgsless mechanism. The left hand side of (2a) vanishes in the SM, but this does not necessitate anomalous top couplings since in principle the contributions of the $b$ and $Z$ - KK modes can cancel each other.

To obtain estimates for the couplings, in [11] the SRs from $W^{+} W^{-} \rightarrow t \bar{t}, W Z \rightarrow t b$ and $Z Z \rightarrow t t$ were solved under the assumption of saturation by the first KK-level and non-degenerate KK-modes. For the example of equal left-and right handed couplings of the KK-modes and non-anomalous zero mode couplings one obtains the estimates

$$
g_{W t B^{(1)}} \approx \frac{g}{2} \sqrt{\frac{m_{t}}{m_{B^{(1)}}}}, \quad g_{t t Z^{(1)}} \approx \frac{\sqrt{3} g}{4} \frac{m_{t} m_{Z^{(1)}}}{m_{W} m_{B^{(1)}}}, \quad g_{t T^{(1)} Z} \approx \frac{g}{2 \cos \theta_{w}} \sqrt{\frac{m_{t}}{2 m_{T^{(1)}}}}
$$

We can check our assumptions in the 5D $S U(2)$ model [6] where the top sector is parameterized by the coefficients $t_{L / R}$ of the BKTs and the $5 \mathrm{D}$ Lorentz violating mass term $\kappa \bar{\Psi}_{t} \partial_{5} \Psi_{t}$. The flavor universal value $t_{L} \sim \pi m_{W} R / \sqrt{3}$ ensures a vanishing $S$ parameter while constraints on the $W t_{R} b_{R}$ vertex and unitarity of $W^{+} W^{-} \rightarrow t \bar{t}$ scattering imply the bound $3.6 \mathrm{TeV}<\kappa / R<32 \mathrm{TeV}$ [6]. These parameters enter the top mass $m_{t} \approx \kappa t_{L} t_{R} /(\pi R)$, the mass of the first bottom KK mode $m_{B^{(1)}} \approx \kappa /(2 R)$ and its coupling constant $g_{W t B^{(1)}}^{L / R} \approx\left(2^{5 / 2} t_{L / R} / \pi^{2}\right) g_{W t b}^{L}$. We find that the the SR $(2 \mathrm{~b})$ is indeed saturated by the first KK-level to leading order in $t_{L / R}$. From the bounds on $\kappa$ we only obtain a weak constraint $g_{W t B^{(1)}}^{L} / g_{W t B^{(1)}}^{R} \sim t_{L} / t_{R} \sim\left(\kappa \pi R m_{W}^{2}\right) /\left(3 m_{t}\right) \sim 0.2-2$ for $R^{-1}=800 \mathrm{GeV}$.

\section{IMPLICATIONS FOR PHENOMENOLOGY}

A simplified Higgsless scenario with a single KK-level and couplings as given in (3) and below (1) can be used to estimate collider signatures in the top sector. The cross sections for $W^{+} W^{-} \rightarrow t \bar{t}$ and $Z Z \rightarrow t \bar{t}$ displayed in figure 2 show a suppression at large $\sqrt{s}$ both in the SM with a Higgs resonance and in the Higgsless scenario while the inclusion of a $Z^{(1)}$ without the $B^{(1)}$ rather destroys the unitarity cancellations present in the SM. In $Z Z \rightarrow t \bar{t}$ no resonance appears in the Higgsless model so the unitarization is entirely due to the $T^{(1)}$ in the $t$ channel and becomes effective earlier on for a lower $m_{T^{(1)}}$.
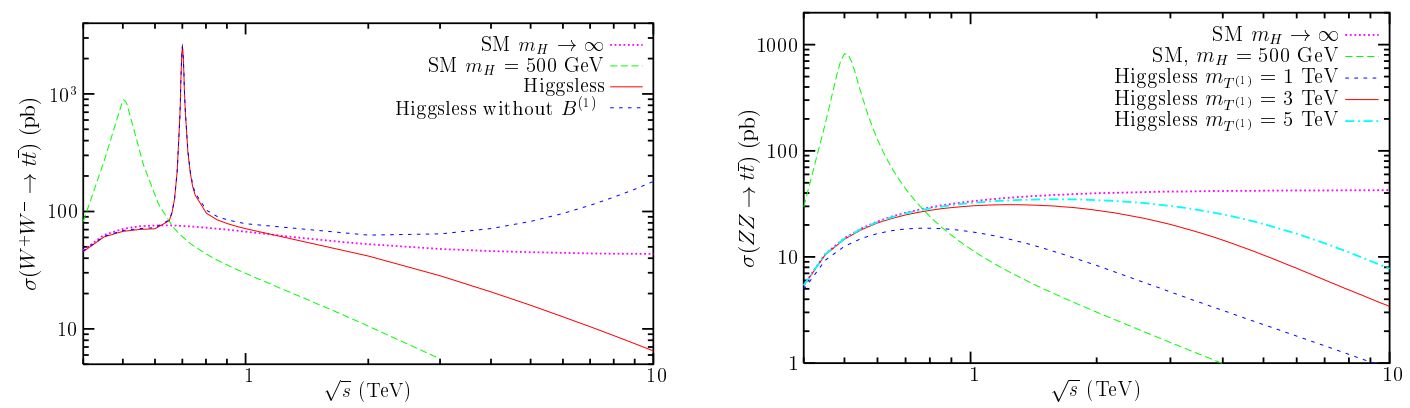

FIGURE 2. (left) Cross section for $W^{+} W^{-} \rightarrow t \bar{t}$ (left) and $Z Z \rightarrow t \bar{t}$ (right) in the SM with $m_{H}=500$ $\mathrm{GeV}$, the SM with $m_{H} \rightarrow \infty$ and the Higgsless scenario with $m_{Z}^{(1)}=700 \mathrm{GeV}$ and $m_{B^{(1)}}=2.5 \mathrm{TeV}$ [11]. 
The couplings to third family quarks affect the phenomenology of the gauge boson KK-modes. From (3) the partial decay width of the $Z^{(1)}$ into top-quarks is estimated as

$$
\Gamma_{Z^{(1)} \rightarrow t \bar{t}} \approx \frac{3 \alpha_{Q E D} m_{Z^{(1)}}^{3}}{16 \sin ^{2} \theta_{w} m_{W}^{2}}\left(\frac{m_{t}}{m_{B^{(1)}}}\right)^{2} \approx \begin{cases}11 \mathrm{GeV} & \left(m_{B^{(1)}}=1 \mathrm{TeV}\right) \\ 1.8 \mathrm{GeV} & \left(m_{B^{(1)}}=2.5 \mathrm{TeV}\right)\end{cases}
$$

which is to be compared to $\Gamma_{Z^{(1)} \rightarrow W W} \approx 13 \mathrm{GeV}$ [10] (here $m_{Z^{(1)}}=700 \mathrm{GeV}$ ).

While a $Z^{(1)}$ resonance in $W^{+} W^{-} \rightarrow \bar{t} t$ at the LHC is overwhelmed by QCD background to $p p \rightarrow t \bar{t} j j$ [15], the associated production with $b$ or $t$ quarks-e.g. in single top production $W b \rightarrow t Z^{(1)} \rightarrow t t \bar{t}$ or top pair production $g g \rightarrow t \bar{t} Z^{(1)} \rightarrow t \bar{t} t \bar{t}$ - has been found promising for $m_{Z^{(1)}}=1 \mathrm{TeV}$ [16], however for a strong coupling to $b$ and $t$ with $\Gamma_{Z^{(1)} \rightarrow t \bar{t}+b \bar{b}}=127 \mathrm{GeV}$ and negligible coupling to the $W$, unlike to Higgsless models.

The production of heavy top quarks in $W-b$ fusion $q b \rightarrow q^{\prime} T$ at the LHC has been studied in the context of Little Higgs models [17] where the relevant coupling is given by $g_{W T b}^{L}=\frac{g}{\sqrt{2}} \frac{m_{t} \lambda_{1}}{m_{T} \lambda_{2}}$. For $\lambda_{1}=\lambda_{2}$, the reach of the LHC has been estimated [17] as $m_{T}=2$ $\mathrm{TeV}$. Comparing to the result analogous to (3) for $g_{W T^{(1)} b}$ [11] one can approximately relate the cross sections in the Littlest Higgs model and in the Higgsless scenario as

$$
\sigma_{\mathrm{HL}}\left(W b \rightarrow T^{(1)}\right)=\frac{m_{b} m_{T}^{(1)}}{m_{t}^{2}}\left(\frac{\lambda_{2}}{\lambda_{1}}\right)^{2} \sigma_{\mathrm{LH}}(W b \rightarrow T) \approx 0.13 \frac{m_{T}^{(1)}}{\mathrm{TeV}}\left(\frac{\lambda_{2}}{\lambda_{1}}\right)^{2} \sigma_{\mathrm{LH}}(W b \rightarrow T)
$$

so the phenomenology of the $T^{(1)}$ will be even more challenging in the Higgsless model. For a light $T^{(1)}$ as in [14], QCD pair production is the dominant signal [17].

To summarize, viable Higgsless models exist $[6,8,14]$, but require a tuning of fermion mass parameters and a separate treatment of the third generation. Unitarity sum rules imply the presence of top and bottom KK-modes. In addition, associated production of the $Z^{(1)}$ with top quarks deserves more detailed studies in realistic models.

\section{ACKNOWLEDGMENTS}

The work described in this talk was supported by the DFG through the SFB/Tr 9 at RWTH Aachen and the Graduiertenkolleg "Eichtheorien" at Mainz University.

\section{REFERENCES}

1. C. Csaki et.al, Phys. Rev. D69, 055006 (2004), hep-ph/0305237.

2. R. S. Chivukula, D. A. Dicus, and H.-J. He, Phys. Lett. B525, 175-182 (2002), hep-ph/0111016.

3. T. Ohl, and C. Schwinn, Phys. Rev. D70, 045019 (2004), hep-ph / 0312263.

4. For a review see C. Csaki, J. Hubisz, and P. Meade (2005), hep-ph / 0510275.

5. G. Burdman, and Y. Nomura, Phys. Rev. D69, 115013 (2004), hep-ph/ 0312247.

6. R. Foadi, and C. Schmidt, Phys. Rev. D73, 075011 (2006), hep-ph/ 0509071.

7. For a review see E. H. Simmons et.al (2006), hep-ph/0606019.

8. H. Georgi (2005), hep-ph/0508014.

9. C. Schwinn, Phys. Rev. D69, 116005 (2004), hep-ph / 0402118.

10. A. Birkedal, K. Matchev, and M. Perelstein, Phys. Rev. Lett. 94, 191803 (2005), hep-ph/ 0412278 . 
11. C. Schwinn, Phys. Rev. D71, 113005 (2005), hep-ph / 0504240.

12. G. Cacciapaglia et.al, Phys. Rev. D72, 095018 (2005), hep-ph/ 0505001.

13. K. Agashe et.al, Phys. Lett. B641, 62-66 (2006), hep-ph/ 0605341.

14. G. Cacciapaglia, C. Csaki, G. Marandella, and J. Terning (2006), hep-ph/ 0607146.

15. T. Han, D. L. Rainwater, and G. Valencia, Phys. Rev. D68, 015003 (2003), hep-ph/ 0301039.

16. T. Han, G. Valencia, and Y. Wang, Phys. Rev. D70, 034002 (2004), hep-ph/ 0405055.

17. For a review see M. Perelstein (2005), hep-ph / 0512128. 